\title{
Democracia y Justicia Social en las Escuelas: Dimensiones para Pensar y Mejorar la Práctica Educativa
}

\author{
Democracy and Social Justice in Schools: Dimensions for \\ Thinking and Improving Educational Practice
}

Guillermina Belavi *

F. Javier Murillo

Universidad Autónoma de Madrid, España

\begin{abstract}
La democracia y la justicia social se implican mutuamente. Esta afirmación, válida para la sociedad en general, lo es también para las escuelas. Con las ideas sobre Justicia Social de Nancy Fraser como marco, este artículo propone cinco dimensiones para avanzar en la Justicia Social y en la Democracia en las escuelas. En primer lugar, Redistribución de oportunidades y beneficios de la educación, como forma de romper el círculo perverso de la desigualdad desde la escuela; Reconocimiento de valores culturales y diversidad social, como segunda dimensión a través de la cual se cuestionan los valores culturales dominantes en la educación y se fomenta el respeto y el aprendizaje de las distintas identidades, perspectivas y estilos de vida de los miembros de la comunidad educativa. La tercera dimensión hace referencia a la Gobernanza escolar, gobernanza basada en la distribución de responsabilidades, la proactividad y la cooperación en redes plurales. La cuarta dimensión es Currículum crítico y participativo y la quinta y última, Cultura escolar democrática. Estas dimensiones invitan a identificar los conceptos abstractos en la forma concreta en que se realizan en las escuelas y, a partir de allí, reflexionar sobre ellos y sugerir prácticas que nos ayuden a mejorarlas.
\end{abstract}

Descriptores: Democracia; Justicia social; Escuela; Gobernanza escolar; Redistribución; Reconocimiento; Currículum democrático; Cultura escolar.

Democracy and social justice are mutually involved. This statement is valid for our society and is also true for our schools. With Nancy Fraser's ideas on Social Justice as a framework, this article proposes five dimensions to improve Social Justice and Democracy in schools. First, Redistribution of opportunities and benefits of education, as a way for schools to break the social inequalities perverse circle; the second dimension is the Recognition of cultural values and social diversity, through which the dominant cultural values in education are questioned, and the different school's member identities, perspectives and lifestyles are respected and learned from. The third dimension refers to democratic school governance, a governance based on responsibilities distribution, proactivity and plural networks cooperation. The fourth dimension is Critical and Participatory Curriculum and the fifth and last one is a Democratic School Culture. These dimensions contribute to identify abstract concepts in the concrete ways in which they are carried out in schools, reflect on them and suggest practices to improve them.

Keywords: Democracy; Social justice; School; School governance; Redistribution; Recognition; Democratic curriculum; School culture.

*Contacto: guillermina.belavi@uam.es

ISSN: $1696-4713$

www.rinace.net/reice/

revistas.uam.es/reice
Recibido: $\quad 13$ de noviembre 2019

$1{ }^{\text {a }}$ Evaluación: 27 de febrero 2020

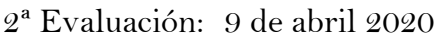

Aceptado: 27 de mayo 2020 


\section{Introducción}

Democracia y justicia social se necesitan mutuamente. Se necesitan en las calles de nuestras ciudades, en el día a día de nuestras comunidades y en nuestras escuelas también. En nuestros centros educativos se necesitan con el particular desafío y responsabilidad de la educación: el de enseñar haciendo más que diciendo. De forma que buscamos dar vida a esta compleja relación en la realidad de nuestras escuelas, colegios e institutos, buscamos guiarnos por ella y transmitirla con el ejemplo; pero también contarla, contar las libertades y dignidades que nos permiten y los desafíos que aún nos quedan por conseguir.

Aunque obligada, la relación entre democracia y justicia social no es sencilla. La filosofa política Nancy Fraser (2000, 2008, 20012) nos ayuda a comprenderla. Sostiene que los principales asuntos de justicia son tres: la redistribución, el reconocimiento y la representación/participación. La primera sería la justicia económica, la segunda la justicia cultural y la tercera la justicia política, esta última la más importante y la condición sine qua non de las anteriores. Para evaluar y saldar las injusticias se necesita un criterio que sea a la vez imparcial y capaz de medir con una vara común asuntos de naturaleza heterogénea: este criterio es la paridad de participación. Por lo tanto, y en sus propias palabras, "la justicia requiere acuerdos sociales que permitan a todos participar como pares en la vida social" (Fraser, 2012, p. 39). Desde esta definición, la redistribución, el reconocimiento y la representación/participación social no son deseables solamente por sí mismas, sino que además resultan indispensables para una participación igualitaria en los asuntos comunes. La participación es fundamental, a su vez, para saldar los problemas de injusticia de diversa índole.

La literatura educativa toma las ideas de Fraser, especialmente su abordaje desde las tres dimensiones de la justicia social, para comprender la educación y las escuelas (p. ej., Abowitz, 2011; Blackmore, 2016; Keddie, 2012; Lim y Tan, 2018; Lynch y Lodge, 2002; Mills et al., 2016; Murillo y Hernández, 2011, 2014; Vincent, 2020). Al poner énfasis en las dimensiones de la justicia social, esta literatura no trabaja directamente sobre la democracia en las escuelas. Sin embargo, también existe literatura educativa que nos brinda pautas para mejorar la democracia en nuestras escuelas (Feito, 2009; Feu et al., 2016, 2017; Fielding, 2012; Moliner et al., 2016; Simó, Parareda y Domingo, 2016; Woods y Woods, 2012). La perspectiva clásica es de Apple y Beane (1999) y sugiere que las escuelas democráticas resultan de iniciativas y esfuerzos en dos líneas de trabajo: "Una es crear estructuras y procesos democráticos mediante los cuales se configure la vida en la escuela. La otra es crear un currículum que aporte experiencias democráticas a los jóvenes" (Apple y Beane, 1999, p. 24). Entre las aportaciones en el ámbito nacional destacamos la del grupo de investigación Demoskole que propone construir escuelas más democráticas a partir de trabajar las dimensiones de gobernanza, habitanza, alteridad y valores, y ethos -virtudes y capacidades- (Feu et al., 2016, 2017; Simó, Parareda y Domingo, 2016).

A partir de esta literatura y teniendo en cuenta los productos del trabajo elaborado en el marco del proyecto del Plan Estatal I+D+i del Gobierno de España "La democracia en las escuelas como fundamento de una educación para la Justicia Social" (Ref. EDU201782688-P), este artículo propone una guía orientativa que nos ayude a reconocer y mejorar la democracia y la justicia social en nuestras escuelas. Para ello desarrolla cinco dimensiones, dando claves para profundizar en cada una de ellas y, a la vez, concretarlas en su relación mutua en la práctica educativa. 


\section{Cinco dimensiones para mejorar la democracia y la justicia social en nuestras escuelas}

No hay recetas ni instrucciones cerradas que nos den claves definitivas, pero la investigación y la experiencia nos ayudan a identificar cinco dimensiones necesarias para para hacer nuestras escuelas más justas y democráticas y, con ello, contribuir a una sociedad más justa: redistribución, reconocimiento, currículum, gobernanza y cultura escolar. La inspiración generada por los trabajos del grupo de investigación Demoskole ha sido clave para esta propuesta, y así lo reconocemos (p. ej., Feu et al., 2016, 2017; Simó, Parareda y Domingo, 2016).

Estas dimensiones están profundamente relacionadas entre sí, tanto que a veces son una perspectiva diferente de atender a lo mismo; pero así consideradas ayudan a identificar prácticas y reflexionar sobre ellas para adaptarlas a cada comunidad escolar y mejorar nuestros centros. Las dos primeras están más relacionadas con la justicia social (redistribución y reconocimiento), mientras que las dimensiones tercera y cuarta están más relacionadas con la democracia (gobernanza y cultura democrática). La dimensión restante es propia de la educación: un currículum crítico y participativo.

1. Redistribución de oportunidades y beneficios de la educación. Los recursos y las oportunidades, distribuidos de manera injusta, impiden que todas las personas puedan partir de bases dignas para construir sus trayectorias. En educación, el problema redistributivo refiere a la inequidad en el acceso, la participación y los resultados, pues no todos los estudiantes están en la misma situación de aprovechar las oportunidades y de alcanzar sus credenciales mínimas (Lim y Tan, 2018; Lingard y Keddie, 2013; Lynch y Lodge, 2002). Las escuelas que buscan interrumpir los círculos viciosos de la desigualdad asignan los recursos del centro según las necesidades del estudiantado, de tal manera que todos puedan disfrutar de una educación de calidad más allá de sus historias personales. De esta manera, las escuelas contribuyen a romper los vínculos entre la pobreza, el bajo rendimiento escolar, el abandono escolar prematuro y la privación económica futura (Keddie, 2012).

2. Reconocimiento de valores culturales y diversidad social. Nuestras sociedades se rigen por una jerarquía de valores culturales que margina y excluye otras formas de ser y de vivir. Desde esta perspectiva, las víctimas de la injusticia no son reconocidas en su igual valor, muchas veces son incluso ignoradas o desprestigiadas. Esta dimensión, por lo tanto, lucha por el reconocimiento de la diferencia para que todos y todas puedan disfrutar de una igual valoración y estima, con indiferencia de sus valores culturales y sus elecciones personales. En las escuelas, la falta de reconocimiento es también un predictor altamente preciso del bajo rendimiento educativo (Keddie, 2012). Por ello, a través de esta dimensión, se cuestionan los valores culturales dominantes en la educación y se fomenta el respeto y el aprendizaje de las distintas identidades, perspectivas y estilos de vida de los miembros de la comunidad educativa.

3. Gobernanza escolar. El concepto de gobernanza (más que el de gobierno) se refiere a modos más flexibles, plurales y dinámicos de llevar adelante la gestión pública que, en sus potencialidades, nos permiten hablar de gobernanza democrática (Subirats, 2009, 2016). Apropiándonos del mismo para la educación (Feu et al., 
2016; Sanizo Rodall, 2011; Smit y Oosthuizen, 2011), esta dimensión promueve la participación, el dinamismo y el trabajo en red en la toma de decisiones y la gestión escolar. Aquí es crucial la participación de familias, la distribución de responsabilidades, la proactividad y la cooperación en redes plurales. Al incorporar a toda la comunidad educativa en las decisiones del centro y promover la organización espontánea, la gobernanza permite un gobierno escolar dinámico y horizontal (Santizo Rodall, 2011).

4. Curriculum crítico y participativo. El currículum es la dimensión exclusiva de la educación. En el conjunto que orquesta con niveles, grados, asignaturas, etc., jerarquiza los esfuerzos escolares y constituye el criterio con el cual se juzgará el proceso educativo. Tiene, por lo tanto, un enorme poder y proyección a futuro. Incumbe a toda la sociedad, pues responde a la pregunta última sobre qué educación queremos y qué sociedad construimos (Carr, 1998; Gutmann, 1987). Esta dimensión da lugar a construir nuevas formas de conocimiento a partir de la crítica de formas hegemónicas heredadas y la valoración de los saberes y experiencias de la comunidad. Los trabajos realizados en torno al currículum integrado son aquí fundamentales (Beane, 1997; Brough, 2012; Gimeno Sacristán, 1991; Guarro, 2002). Pero la democracia es también un contenido curricular en sí misma, pues es una conquista histórica y sólo se mantendrá viva y mejorando si la aprendemos como tal (Carr, 1998; Ruitemberg, 2009).

5. Cultura escolar democrática. Entendida como el conjunto de normas, valores, creencias, tradiciones y rituales que se van construyendo a lo largo del tiempo, y son compartidos por la comunidad educativa. Los elementos de la cultura escolar se unen en un todo profundo que configura las expectativas y las asunciones básicas de los participantes, guía las actividades del personal escolar y de los estudiantes (Hinde, 2005). Es un elemento clave, pues muchos intentos de promover la participación o la equidad en las escuelas fallan porque no permean en la cultura del grupo. Es que la democracia no es (al menos en primera instancia) una técnica o procedimiento para tomar decisiones y escoger la forma de organizarnos. Tampoco la justicia social es una distribución mecánica de beneficios ni un respeto impuesto a la diversidad. Son más que ello, se alimentan de los significados asumidos por la comunidad escolar y construidos en sus interacciones cotidianas.

Estas dimensiones nos ayudan a trabajar la democracia y la justicia social en sus relaciones mutuas. En tanto asuntos de justicia, la redistribución, el reconocimiento y la representación/participación (que se juega aquí en la dimensión de gobernanza) son deseables por sí mismas, pero también son necesarias para avanzar hacia la paridad de participación en la escuela. Y esa paridad de participación, es decir, la posibilidad de todos de participar en términos de igualdad con sus pares, es necesaria para dejar al descubierto asuntos de justicia que estén siendo pasados por alto. Así, por ejemplo, una representación desigual en la toma de decisiones escolares puede mejorarse si las personas de distintas culturas hacen oír su voz y reclaman los espacios que legítimamente les corresponden. Y a la inversa, la representación de las personas de distintas culturas en las instancias formales de decisión en la escuela favorece el reconocimiento de estos colectivos y contribuye a que sus miembros participen en igualdad de condiciones (igualdad de estatus) con sus pares. 
Cada uno a su manera, el currículum y la cultura escolar democrática atraviesan las otras dimensiones. La redistribución, el reconocimiento y la participación configuran y constituyen un currículum crítico y participativo. Cada una de las dimensiones, a su vez, es genuina en tanto se corresponda con significaciones compartidas, normas, valores y patrones de relación en la escuela.

\section{Redistribución de oportunidades y beneficios de la educación}

La dimensión redistributiva tiene que ver fundamentalmente con la justicia social, especialmente con una perspectiva que enfatiza la equidad en la disposición de bienes mínimos, derechos y oportunidades básicas. Según esta visión, la justicia consiste en una distribución equitativa que permita una vida digna a todas las personas y sostiene que la igualdad de oportunidades es una falacia si no existe una igual distribución de bienes, derechos y/o capacidades que permitan aprovecharlas. En nuestra área, esta dimensión obliga a reconocer que la sociedad no es equitativa y que, por lo tanto, los estudiantes no están en la misma situación de aprovechar los beneficios de la educación. Las escuelas deben esforzarse para lograr una distribución más equitativa de las oportunidades y beneficios educativos (Apple y Beane, 1999; Keddie, 2012; Wang, 2016).

Los defensores de la redistribución centran su atención en el aspecto socioeconómico y sostienen que las diferencias sociales son los resultados de la estructura de una economía política injusta (Fraser, 2008). En su definición, la distribución tiene una referencia filosófica que remite a paradigmas normativos elaborados por la filosofía moral y una referencia política que la relaciona con reivindicaciones de actores políticos y movimientos sociales. En términos filosóficos, esta dimensión se nutre del liberalismo igualitarista que hacia finales del siglo XX realiza un esfuerzo teórico importante para conciliar la libertad individual con el igualitarismo de la socialdemocracia (Rawls, 1971; Dworkin, 2006). En términos de luchas sociales y acción histórica, esta dimensión remite a las reivindicaciones de clases y las luchas por un cambio en la estructura económica que mejore las condiciones de igualdad material.

En educación también apelamos a la dimensión redistributiva para que la igualdad de oportunidades sea posible. Desde esta perspectiva, es posible afirmar que, en esencia, la injusticia es un problema de inequidad en el acceso, la participación, las condiciones y los resultados de la educación (Keddie, 2012; Lim y Tan, 2018; Lingard y Keddie, 2013; Lynch y Lodge, 2002; Power, 2012; Wang, 2016). De manera que esta dimensión reconoce, por un lado, que las escuelas no son equitativas en la distribución de los servicios que presta $y$, por el otro, que los estudiantes no están posicionados de manera equitativa en su capacidad para aprovecharlos (Keddie, 2012).

En términos sociales, la dimensión distributiva en educación tiene relación fundamental con la inequidad económica, pues los estudiantes de familias de menores recursos económicos no tienen posibilidad de acceder y participar en las mismas condiciones que sus pares privilegiados. La desigualdad socioeconómica es aquí la causa generativa del problema educativo. Aun así, el problema distributivo en educación no es puramente un asunto de clase. Muchas injusticias (como aquellas que sufren personas con capacidades distintas, diferente cultura o religión, origen nacional, elección sexual o identidad de género) también se manifiestan como problemas de distribución, pero la causa generativa 
de estas injusticias es una falta de reconocimiento. Estas personas son subordinadas en la distribución de recursos y beneficios a raíz de sufrir un estatus desigual (Lynch y Lodge, 2002).

La dificultad inicial para aprovechar las oportunidades educativas se proyecta en el futuro de los estudiantes más pobres y de todos aquellos perjudicados por una distribución desigual, ya que la educación determina en gran medida las credenciales de empleo y el subsecuente acceso al mercado laboral (Mills y Gale, 2010). Estos estudiantes, por lo tanto, tendrán menores posibilidades de acceder a un puesto de trabajo dignamente retribuido y de insertarse socialmente en condiciones de justicia económica. Además de distribuir los recursos bajo el criterio de equidad para aprovechar las oportunidades educativas, esta dimensión también se ocupa de que todos y todas alcancen una educación de calidad. Se trata de que todos y todas tengan la oportunidad de disfrutar de los beneficios educativos y de las credenciales que equiparan las condiciones en el mercado laboral.

En términos del sistema educativo, la dimensión distributiva está relacionada con iniciativas que asignan fondos y recursos adicionales a grupos de estudiantes sobre la base de la necesidad económica o que distribuyen recursos materiales a las escuelas situadas en contextos más desafiantes. También incluye iniciativas que asignan personal docente mejor capacitado a estas escuelas, e incluso en un número mayor. Pero aquí nos interesa el papel de los centros escolares. De hecho, la tarea de las escuelas también es fundamental para romper la relación causa-efecto entre la pobreza, el bajo rendimiento escolar, el abandono escolar prematuro y la privación económica futura (Keddie, 2012).

Las escuelas que atienden a la redistribución de oportunidades y beneficios educativos se ocupan de asignar los recursos del centro según las necesidades del estudiantado de tal manera que todos puedan acceder y aprovechar de la educación que se brinda y alcanzar una formación de calidad más allá de sus historias personales. Prestan atención a que se cumplan las condiciones mínimas de calidad de vida del estudiantado, ya que no puede uno concentrarse con hambre, con sueño o sin los materiales adecuados. De esta manera, estructuran la asignación de recursos de la escuela según las condiciones iniciales de sus estudiantes, disponiendo más recursos para aquellos que tienen dificultades socioeconómicas, que están excluidos o en riesgo de exclusión. Las escuelas, por lo tanto, organizan y asignan los recursos materiales y didácticos de manera equitativa según las necesidades de los estudiantes.

Muchas veces los problemas de raíz distributiva exceden las posibilidades del centro. En estos casos, las escuelas participan de programas sociales de alimentación o contención, incluso se coordinan con organismos sociales para que los profesionales adecuados sigan de cerca y de manera personalizada la situación de los niños y niñas en riesgo. Es fácil reconocer aquí el vínculo con la gobernanza escolar, pues la manera de hacer frente a la situación de injusticia que sufren estos estudiantes es construir redes colaborativas con entidades del barrio y la ciudad. Teniendo en cuenta las relaciones que construyen estas escuelas "por fuera" del centro para mejorar la dimensión redistributiva, destacamos también las luchas por mejorar las condiciones de trabajo del equipo docente, para que puedan atender adecuadamente a los niños y niñas que más lo necesitan: aumentando el personal en escuelas en contextos desafiantes y valorando la formación para poblaciones en riesgo de exclusión. 
Estas escuelas también se esfuerzan por redistribuir oportunidades educativas en casos de problemas que no tienen una raíz directamente socioeconómica (Lynch y Lodge, 2002), de modo que también adaptan la infraestructura escolar y distribuyen recursos materiales, didácticos y apoyo profesional según las peculiaridades de los estudiantes, atendiendo a quienes tienen dificultades para aprovechar en condiciones de igualdad las oportunidades educativas (discapacidad, dominio del lenguaje, etc.). En este sentido, el trabajo de las escuelas es similar a lo que Feu y colegas $(2016,2017)$ reúnen bajo la dimensión de habitanza.

Además de equiparar las condiciones de acceso, las escuelas trabajan bajo esta dimensión para que todos y todas consigan la misma calidad en los resultados educativos. En este sentido, resulta crucial asistir a los estudiantes marginados para que alcancen el mismo parámetro de logros educativos que sus contrapartes privilegiadas (Keddie, 2012), de manera que puedan educarse como pares y eventualmente acceder a la sociedad de la misma manera que cualquier otro estudiante lo haría. Para ello, las escuelas fomentan que todos los estudiantes, especialmente aquellos en riesgo de exclusión, puedan acceder al capital cultural dominante a través, por ejemplo, de diseñar tareas que resulten intelectualmente exigentes y a la vez motivantes (Lim y Tan, 2018). Además, un aspecto común entre maestros y maestras que se preocupan por la redistribución es que creen en las capacidades de los estudiantes para el éxito académico. Creen en sus estudiantes, y así les transmiten una alta expectativa y la confianza de que aquellos menos favorecidos se harán lugar al igual de bien que sus homólogos privilegiados (Mills y Gale, 2010).

De esta manera, las escuelas brindan herramientas a los estudiantes menos privilegiados para que puedan obtener un mayor acceso a los beneficios materiales del mundo social y buscan desestabilizar las estructuras de clases sociales que generan explotación económica, marginalización y privación para algunos grupos (Lingard y Keddie, 2013; Keddie 2012).

\section{Reconocimiento de valores culturales y diversidad social}

Así como muchas injusticias se generan en la estructura socioeconómica, otras tienen su origen en el campo sociocultural y simbólico. Suceden en la interrelación entre personas y cristalizan en normas culturales e institucionales que restringen o censuran distintas formas de comprender la vida (Fraser, 2000, 2008). Estos patrones dominantes establecen como "normal" una visión del mundo que deja fuera otras culturas y otras formas de ser y de entender la vida y la propia identidad. Lo mismo sucede en nuestras escuelas, donde las desventajas que experimenta el estudiantado no provienen exclusivamente de una distribución injusta de recursos, pues la falta de reconocimiento es también un predictor muy preciso del bajo rendimiento educativo (Keddie, 2012).

La dimensión del reconocimiento también se explica desde la filosofía y desde la acción política. Enraizado en los trabajos de Hegel, el término refiere a una relación recíproca ideal entre sujetos en la que cada uno ve al otro, a la vez, como su igual y como separado de sí. Se estima que esta relación es constitutiva de la personalidad: uno se convierte en sujeto individual sólo en tanto reconoce a otro sujeto y es reconocido por él (Honneth, 1996; Taylor, 1994; Young, 2011). Desde este punto de vista, las víctimas de la injusticia se distinguen por el respeto, estima y prestigio de menor entidad que disfrutan en relación 
con otros grupos de la sociedad y en sus luchas reivindican que la diferencia sea reconocida y valorada. El caso clásico de este tipo de injusticia es el del grupo étnico de bajo estatus que es señalado por los patrones de valor cultural dominantes como diferente y menos valioso; pero también los grupos racializados, los grupos feministas o aquellos a favor de la diversidad sexual se movilizan por un mundo más justo comprendido desde esta perspectiva (Fraser, 2008).

El problema de la falta de reconocimiento es que cuando las personas tienen formas de ser y conocer diferentes a las dominantes quedan sujetas a una jerarquía de valores culturales que no es la suya, que les es extraña y muchas veces incluso hostil. La falta de reconocimiento se manifiesta muchas veces como invisibilización, pero también como subordinación o denigración (Fraser, 2000; Young, 2011). La invisibilización sucede cuando se niega rotundamente la existencia de la diferencia y se vive como si no existiese esa otra forma de ser o comprender el mundo. La subordinación sucede cuando las personas no son reconocidas adecuadamente según sus contribuciones sociales. Finalmente, la denigración sucede cuando las formas de vida diferentes son difamadas o mensopreciadas, tanto en representaciones públicas como en la vida cotidiana: sus identidades se tergiversan, son estereotipadas y se las define a partir de características negativas. Dado que las relaciones interpersonales son constitutivas de la subjetividad, muchas veces las propias víctimas de la injusticia internalizan estas visiones negativas y se juzgan a sí mismas desde patrones culturales que les son hostiles.

El problema de la falta de reconocimiento también se manifiesta en los distintos niveles de la educación. El sistema educativo se estructura por un criterio meritocrático y opera en una cultura dominante donde el éxito se define de manera limitada según valores hegemónicos (Power y Frandji, 2010). En este contexto, la segregación escolar manifiesta de manera cruda las injusticias por falta de reconocimiento. Las escuelas que concentran grupos de población marginada suelen tener malos resultados y sufren con ello las tres dimensiones de reconocimiento erróneo: los éxitos que logran, que podrían ser muy importantes en relación con el perfil sociocultural de su estudiantado, no se valoran adecuadamente, de manera que los esfuerzos de estas comunidades escolares se vuelven invisibles en las clasificaciones crudas. Además, las clasificaciones mismas constituyen una falta de respeto hacia estas comunidades escolares (Power y Frandji, 2010).

Como nuestras sociedades, la educación también se rige (por defecto) por parámetros jerárquicos e inequitativos de reconocimiento cultural, de manera que en las escuelas también se invisibiliza, subordina y denigra. Así lo demuestra el trabajo de Lynch y Lodge (2002) en el que investigan las formas concretas en que ocurre la falta de reconocimiento. Según sus resultados, un ejemplo típico de invisibilización sucede ante la orientación sexual de las personas. La subordinación o reconocimiento erróneo es sufrido especialmente por las minorías raciales y étnicas, las personas con discapacidad o habilidades diferentes y las minorías religiosas. Y los estereotipos y la denigración de la diferencia provocan con frecuencia la exclusión de los estudiantes con discapacidades de una plena participación en la educación (Lynch y Lodge, 2002). El problema se agrava dado que la homogeneidad con la que se construyen los patrones de "normalidad" en las escuelas lleva a que los miembros de los grupos no reconocidos se vean a sí mismos a través de esta lente e internalicen a menudo los estereotipos a los que están sometidos.

Además, como este tipo de injusticia muchas veces es sufrido por grupos minoritarios, sucede que suelen ser pocos niños y niñas que pertenecen a estos grupos en la escuela, de 
manera que la falta de reconocimiento no se asume como una injusticia social, sino como una característica personal, lo que hace más difícil abordarla como un problema que trasciende las biografías individuales. El resultado es que, en el mejor de los casos (cuando no hay invisibilización, exclusión, marginación o denigración), la persona afectada pasa a ser más un sujeto de caridad que de derecho (Fraser, 2012). Tal es así que se ha comprobado incluso una falencia en el vocabulario de los estudiantes que le permita hablar abiertamente y discutir sobre las diferencias sociales y culturales (Lynch y Lodge, 2002). Les resulta difícil tratar sobre la diversidad y expresar sus visiones sin caer en prejuicios y, cuando se les pregunta por ello, las respuestas tienden a variar entre la negación y el silencio (generalmente ante la diversidad sexual), la hostilidad (mayormente hacia inmigrantes), la cautela (ante diferencias religiosas) y la lástima (en el caso de problemas de discapacidad).

Identificar esta dimensión ayuda a mejorar las prácticas que aumentan el reconocimiento y fomentan la igualdad de estatus entre las personas. Para empezar, las escuelas que atienden a esta dimensión saben que no hay valores "normales" sino valores hegemónicos (Keddie, 2012). Por lo tanto, se preguntan desde qué patrones se relacionan y educan, es decir, cuáles son los criterios que rigen las relaciones entre los miembros de la comunidad educativa y cuáles son los valores predominantes que fomenta la educación que se imparte en el centro. El problema fundamental no es la prevalencia de un criterio o de unos valores sobre otros (lo que resulta difícil de evitar), sino el hecho de que se silencie la diferencia y se genere una idea homogénea de "normalidad" que censura otras visiones del mundo (Sapon-Shevin, 2013).

Las comunidades educativas que trabajan esta dimensión reconocen y respetan la diversidad en su experiencia cotidiana. Aprenden de todos y todas creando espacio y dando lugar para que la diversidad se manifieste, viviendo con ella, lidiando con sus dificultades y disfrutando de sus alegrías. Saben que la inclusión y el respeto mutuo no se aprende en abstracto, sino que se internalizan a través de las prácticas cotidianas y el entrenamiento desde una perspectiva crítica y empática (Lynch y Baker, 2005).

Los maestros y maestras que fomentan la justicia como reconocimiento saben que los estudiantes a menudo provienen de entornos menos favorecidos o minoritarios y poseen un capital cultural que es diferente (a veces incluso contrapuesto) al que promueve el sistema educativo. Por lo tanto, se esfuerzan por debilitar las jerarquías de estatus y los patrones de valor cultural homogéneos y privilegian las experiencias y las fuentes de conocimiento que los grupos menos aventajados aportan a la escuela. Utilizan los saberes y los marcos de referencia de sus estudiantes para crear experiencias y oportunidades que resulten relevantes para alcanzar los estándares educativos, pero también valoran los conocimientos y la visión del mundo de las minorías culturales por sí mismos, aprendiendo de ellos y cuestionando las visiones dominantes (Gay, 2013; Lim y Tan, 2018).

Estos maestros y maestras también atienden a las asignaturas y a las inteligencias que son menos reconocidas y que tienen menor estatus social que aquellas valoradas en los rankings internacionales y los exámenes nacionales. Crean las oportunidades para que los estudiantes demuestren sus habilidades y conectan con su vida fuera de las escuelas (Lim y Tan, 2018). Por último, la interculturalidad y el principio de inclusión están presentes en los valores del centro y en el currículo, la pedagogía y los sistemas de evaluación. Esto significa que tienen importancia como tópicos, pero también que se incluye a los miembros 
de los grupos minoritarios y marginados en el diseño de programas educativos y de convivencia en las escuelas (Lynch y Baker, 2005; Lynch y Lodge, 2002).

\section{Gobernanza escolar democrática}

Las escuelas que trabajan por ser más justas y democráticas atienden a la organización escolar y al gobierno del centro: su estructura y funcionamiento, el ejercicio de los distintos roles y funciones, los mecanismos privilegiados para la toma de decisiones y los modos de gestión escolar. Pero la gobernanza escolar, aunque hace referencia a estos aspectos, no atiende tanto a normas y procedimientos estatuidos ni a organigramas de posiciones fijas de poder, sino al modo dinámico y vivo en que se construyen las decisiones. La gobernanza nos remite a las funciones sociales o procesos en torno a la definición de los asuntos que se desarrollan de diversas maneras y por una diversidad de actores y no a estructuras en sí mismas.

Con las transformaciones tecnológicas y culturales de los últimos años, cambió la manera en que se toman las decisiones y se ejerce la autoridad. No se trata ya de definiciones basadas en jerarquías estatuidas, en la especialización y en procedimientos burocráticos, sino que actores públicos y privados influyen a través de diversos mecanismos en los asuntos públicos. La complejidad se instaló como elemento intrínseco a los procesos políticos y el concepto de gobernanza se asume con la constatación de este cambio en la forma de gobernar (Aguilar Villanueva, 2006). El concepto reconoce que el poder político se encuentra disperso entre una gran diversidad de actores y que las decisiones de gobierno son el resultado de distintas influencias y mecanismos que no son siempre formales ni fijados por la autoridad competente. La gobernanza no es de por sí un concepto positivo para la democracia; de hecho, muchas veces los mecanismos de influencia responden a redes de intereses y cuotas diferenciales de poder y no a criterios de participación igualitaria. Sin embargo, la gobernanza habilita la utilización de los nuevos mecanismos con criterios democráticos y a favor de una participación genuina incorporando estas prácticas de coordinación a través de redes, fórums deliberativos y participación colaborativa (Subirats, 2009).

La gobernanza democrática asume que el proceso de gobierno no responde a lugares fijos de autoridad, sino que es el producto espontáneo y en cierto modo imprevisto de una interacción compleja entre actores de naturaleza muy variada (Subirats, 2009). Pero al ser democrática, pone énfasis en la participación de actores diversos en el marco de redes plurales y permite que la complejidad habilite procesos de aprendizaje comunitario. Asume que el conocimiento se encuentra disperso, que la autoridad es un concepto difuso que sólo puede desarrollarse en un marco de diálogo y negociación permanente, que los recursos para el desarrollo eficaz están distribuidos en una multiplicidad de sujetos y, finalmente, que las intervenciones provocan una serie de efectos difíciles de prever, de manera que las consecuencias sirven al aprendizaje colectivo

Joan Subirats (2016) propone aplicar esta nueva lógica a la gobernanza escolar para que el bien común, lo grupal y lo comunitario sean a la vez el objetivo y la nueva lógica de la educación. Aboga por un tipo de gobernanza mucho más horizontal, abierta, participativa y auto-organizada de los claustros y las direcciones, y con un tipo de lógica democrática y de lo común aplicada a contenidos, didácticas y evaluaciones. A través de procesos 
continuos de innovación social las escuelas pueden experimentar nuevas racionalidades y formas organizativas con mucha más relación con las familias, la comunidad y el territorio.

De manera que, sin desconocer los marcos institucionales ni las responsabilidades individuales, la gobernanza escolar democrática plantea nuevas formas de coordinación y colaboración que fomentan la participación en red y los procesos de organización espontánea de la comunidad escolar y permiten un gobierno escolar más dinámico y horizontal (Santizo Rodall, 2011). Los lugares de autoridad escolar se asumen, pero el poder no se concentra. Las decisiones se abren a la participación de toda la comunidad educativa, delegando responsabilidades y promoviendo las iniciativas, y se fomenta la cooperación y la coordinación en la gestión cotidiana del centro. La gestión cotidiana se desvincula de lo personal y se concibe como una lógica de organización, una lógica de lo común. La gestión participativa de las escuelas involucra a todos sus participantes y los límites entre escuela y comunidad se vuelven más difusos.

El mayor reto de las comunidades educativas es que la participación sea genuina. Las iniciativas para promover la participación y la colaboración fallan cuando pretenden imponerse sin construir el consenso y sin un interés arraigado en los participantes (Mabovula, 2009; Mncube y Mafora, 2013; Smit y Oosthuizen, 2011). Las escuelas que logran mantener la dinámica en la gobernanza democrática adaptan las sugerencias a partir de las peculiaridades del contex to en que se aplicarán, teniendo en cuenta su historia y su cultura (Mncube y Mafora, 2013; Olivo Pérez, Alaníz Hernández y Reyes García, 2011). Pero podemos aun así nombrar algunas prácticas que fomentan la gobernanza escolar democrática.

El ejercicio del liderazgo distribuido es una buena manera de fomentar la gobernanza escolar democrática ya que asume los lugares tradicionales de autoridad no como un atributo personal de poder sino como espacios de coordinación. La tarea de los líderes es conocer los intereses y las habilidades de los miembros de la comunidad, fomentar los encuentros y la participación de todos en la definición de los asuntos y en la construcción de lo común. Los lugares fijos de autoridad se abren a liderazgos compartidos por la comunidad en su conjunto, cambiantes según el asunto y el momento. En este ejercicio, el gobierno se transforma en un asunto más horizontal y los miembros de la comunidad educativa tienen la oportunidad de desarrollar su capacidad de liderazgo, de estimular y ver reconocido su talento (Murillo, 2006). El liderazgo distribuido fomenta el dinamismo y la participación en las actividades formales e informales, permite identificar las posibilidades emergentes y llevar adelante actividades planificadas de antemano.

Un liderazgo ejercido en múltiples niveles y cambiante según los asuntos requiere redes de colaboración para organizar un sistema que se vuelve complejo (Jones et al., 2014). Aquí también están presente las formas de gobernanza democrática en la escuela, pues se fomenta la gestión en red de los centros educativos y se crean formas de legitimidad compartida para coordinar la complejidad creciente. Los asuntos cotidianos se resuelven de manera que no quedan atados a burocracias o a la espera de la atención y decisión de una autoridad formal, sino que, como producto de una comunicación y coordinación fluida, la gestión en red permite identificar y resolver de manera eficaz y colaborativa los asuntos escolares.

La gestión en red genera espacios para la participación y la colaboración significativa de las familias, personal no docente y los propios estudiantes. Su flexibilidad, al contrario de las estructuras y organigramas rígidos, permite ajustarse a las dinámicas propias de la 
comunidad y de los ciclos escolares. A su vez, fomenta que cada persona aporte a lo común según su propia impronta y con ello, a través de un compromiso mayor hacia los asuntos de la escuela, se obtienen mejores logros de los que podrían conseguir con gestiones rígidas y principios restrictivos de participación.

La gobernanza escolar democrática permite combinar las decisiones formales e informales, los espacios institucionalizados y no institucionalizados de decisión (Feu et al., 2016, 2017). Aunque la gestión en red cambia la manera de decidir, no desacredita los espacios formales de deliberación y decisión, donde aún se tratan los asuntos estratégicos y generales de la escuela. De hecho, ayuda a que estos órganos de gobierno sean permeables a las inquietudes y voluntades de la comunidad educativa, que los asuntos que llegan hasta allí sean puestos en agenda por una comunicación permanente y la participación compleja (desde distintos espacios) de todos los miembros. Los espacios para tratar asuntos comunes, deliberar y tomar las decisiones están abiertos a la participación efectiva de toda la comunidad educativa. De esta manera, a partir de los mecanismos de gobernanza, los gobiernos escolares se vuelven horizontales y deliberados.

Esto implica también entablar relaciones estrechas y redes de colaboración de la escuela con su barrio y la comunidad en general. La gobernanza escolar democrática promueve la coordinación entre escuelas y autoridades educativas y las relaciones entre todos los actores de la educación en general, gubernamentales y no gubernamentales, escolares y extraescolares (Sanizo Rodall, 2011). Lo que prima es una lógica de lo común y una organización de la participación según la motivación y las habilidades de los participantes. $\mathrm{Al}$ ampliar la participación, desvincular el gobierno de los asuntos comunes de posiciones fijas de poder, se amplían los espacios de acción, las tareas y los límites con la comunidad se hacen más difusos. La escuela es parte de la comunidad general y en su tarea se vincula con espacios, personas y asociaciones que no son directamente educativas, pero que contribuyen a la tarea. Esto permite colaboraciones con el barrio y la comunidad que son mutuamente enriquecedoras.

\section{Currículum crítico y participativo}

El currículum es una dimensión característica de la educación. Organiza las actividades escolares y legitima sus funciones, de manera que los esfuerzos por avanzar en la justicia social y en la democracia se desvanecerán si no les damos entidad en el currículum.

En esencia, constituye la respuesta que dan las escuelas a las preguntas "¿qué debemos enseñar?”, “¿cómo hemos de hacerlo?”, “¡cómo y qué evaluamos para conocer si se ha producido el aprendizaje?” Así, el currículum organiza las prioridades en la escuela, jerarquiza saberes, prioriza actividades y determina los requerimientos para dar cuenta del aprendizaje. En el conjunto que orquesta con niveles, grados, asignaturas, etc., constituye "el estándar respecto del cual se juzgará lo que se considerará el éxito y el fracaso, lo normal y lo anormal, lo satisfactoria o insatisfactoria que es la institución escolar, quiénes cumplen y quiénes no lo hacen” (Gimeno Sacristán, 2010, p. 24). Las decisiones en torno al currículum, por lo tanto, proporcionan el criterio oficial para definir quién avanza en la senda educativa y quién no, por ello su concreción particular es siempre un tema de controversia, pues sus consecuencias se proyectan muy lejos de la institución escolar hacia el orden social y el destino de las personas. 
La pedagogía crítica realizó una gran aportación al investigar las relaciones entre currículum y poder, preguntándose quién define el plan de estudios y qué implicancias tiene el conocimiento oficial en el mantenimiento de los privilegios sociales y en la reproducción de las desigualdades (Apple, 2014, 2017; Giroux, 2001). Pero esta corriente no fue la primera ni es la única en alentar a la comunidad educativa a cuestionar las formas heredadas y construir nuevo conocimiento a partir de sus propias experiencias. En efecto, hace más de un siglo John Dewey (1916/2004) había argumentado a favor de aproximaciones más democráticas. Sostenía que el plan de estudios debía construirse en colaboración y amplió su definición al introducir la experiencia del alumno. A su juicio, las experiencias del niño o la niña proporcionaban significado al plan de estudios. También para Paulo Freire $(1970,1993)$ la experiencia de los educandos era fundamental en la construcción del conocimiento. Argumentaba que los aprendizajes debían ser relevantes para los educandos y que el proceso de alfabetización debía partir de sus conocimientos y sus propias experiencias.

La investigación y la práctica educativa generadas a partir de estas influencias nos permiten sintetizar algunas ideas sobre la construcción del currículum. En primer lugar, enfatizamos que, al igual que ocurre con la justicia social (Murillo y Hernández, 2014), un currículum democrático requiere plantearse en la democracia y para la democracia (Apple, 2018, 2019; Carr, 1998). Esto es, definirse de manera democrática y plantear contenidos democráticos. Como contenido, el currículum tiene el objetivo de promover una conciencia histórica y crítica en los estudiantes, para que comprendan que la democracia es el resultado de luchas pasadas que demandaron una mayor igualdad política, y que las instituciones democráticas son conquistas históricas que tenemos el deber de reformar constantemente para seguir avanzando hacia los ideales que le dieron origen (Carr, 1998; Ruitenberg, 2009).

El diseño de un currículum democrático es un asunto que involucra a toda la comunidad. La pregunta acerca de qué educación queremos es la pregunta sobre qué sociedad queremos (Straume, 2016). La respuesta, por lo tanto, no puede ser producto de una discusión cerrada entre individuos o grupos: así como en una democracia nadie puede detentar autoridad absoluta sobre la sociedad, tampoco puede hacerlo sobre la educación. El plan de estudios, por lo tanto, está formulado sobre la base de una discusión generalizada acerca del papel de la educación en la configuración de la sociedad y será legítimo sólo en la medida en que no prive a nadie de la oportunidad de participar en su definición (Carr, 1998; Englund, 2015; Gutmann, 1987; Hopkins, 2014).

Además de la participación comunitaria en su definición general, es importante un currículum que asuma el conocimiento como un asunto integral y relacionado con nuestra realidad. El abordaje del currículum integrado nos da pautas en este sentido, pues permite establecer conexiones entre los distintos temas de una asignatura, pero también entre distintas asignaturas. Supera las fronteras arbitrarias dispuestas entre disciplinas e integra distintos contenidos en unidades coherentes, lo que permite comprender las relaciones que integran el conocimiento y no asumirlo como compartimentos estancos de saberes cristalizados (Gimeno Sacristán, 1991; Riddle y Apple, 2019; Torres, 2006). La potencialidad del currículum integrado para la democracia se desarrolla cuando va de la mano con una metodología adecuada. Esto nos remite a metodologías que fomenten procesos de enseñanza aprendizaje cooperativos (Guarro, 2008), pero también a un proceso de construcción participativa entre docentes y estudiantes que los lleve a conocimientos relevantes con arraigo en sus experiencias cotidianas. De esta manera, su 
concreción en el aula también es un proceso democrático, una creación conjunta entre el profesorado y los estudiantes (Beane, 1997, 2013; Brough, 2012).

El proceso mismo de enseñanza se inicia con preguntas que surgen de su día a día, de sus vivencias, y que se asientan en lo que les despierta interés. Se trata de que los temas que se abordan adquieran para los y las estudiantes un significado personal. Sin duda, su conocimiento previo es relevante y es utilizado como un punto de partida inicial sobre el que se construye la ruta de aprendizaje (Hytten, 2015; Kumashiro, 2015). A partir de allí, el papel del profesorado es plantear preguntas que definirán el trayecto: qué es lo que ya saben sobre el tema, qué les gustaría averiguar, cómo podrían ordenar la información disponible, qué habilidades les parece que requerirán, cómo presentarían y evaluarían su aprendizaje (Ayers et al., 2016; Brough, 2012). De esta manera, el maestro o maestra puede ampliar y ordenar el proceso planteando preguntas e incorporando experiencias.

La construcción conjunta del currículum cambia el equilibrio de poder entre estudiantes y docente, pues la responsabilidad pasa a ser compartida. El papel particular del profesorado es guiar la creación conjunta del plan de estudios. La tarea exige generar preguntas hábiles que despierten la curiosidad de los estudiantes, ayuden a ampliar el pensamiento, crear debate, empoderar, alentar a asumir riesgos, generar el espacio para que el estudiantado entrene la habilidad de plantear sus preguntas críticas (Bergmark y Westman, 2015). Generar un diálogo crítico consiste tanto en entrenar habilidades de lógica y análisis de argumentos como en enfrentarse a dilemas morales cotidianos que constituyen las verdaderas dificultades de la democracia: consideraciones de lo correcto y lo incorrecto (que no tienen respuesta definitiva mediante un análisis lógico) y análisis de los problemas sociales y morales desde perspectivas profundas, múltiples y variadas. Los problemas cotidianos de una democracia son complejos porque son reales; no porque estén formulados en argumentos sofisticados, sino porque implican comprender y tomar decisiones morales sobre la vida que afectan a los demás (Lim, 2011).

En el proceso de construcción participativa del currículum surgen nuevos temas que complementan los contenidos previstos. Se trata de razonar de manera conjunta sobre los problemas que constituyen el día a día en las escuelas y en la comunidad. La investigación, de hecho, evidencia que los diálogos más efectivos para el proceso de aprendizaje no tienen agenda y se generan en torno a asuntos particulares que no habían sido planteados con el objetivo de conseguir un logro predeterminado, sino porque los maestros realmente buscaban y se interesaban por las opiniones de los estudiantes sobre el tema (Ayers et al., 2016; Brough, 2012). Sostiene además que la resolución de problemas y cuestiones de los docentes plantean para pensar en voz alta también resultan efectivas, como cuando los maestros preguntan en voz alta cómo encarar un asunto, o qué herramientas utilizar para resolver alguna cosa y dan el lugar a los estudiantes para participar. Al actuar según las propuestas de los estudiantes, se les alienta a ofrecer propuestas más innovadoras y ambiciosas.

El resultado que se busca en estos casos no es un mero aprendizaje teórico, sino que se considera a los estudiantes agentes de cambio social y el resultado de los procesos de enseñanza y aprendizaje buscado es el compromiso y la acción (Bergmark y Westman, 2015; McLaren et al., 2010). Al diálogo crítico se le suma la praxis. El diálogo entre estudiantes y profesorado implica la acción reflexiva (individual y colectiva) y produce un cambio simultáneo en el grupo y en las propias personas, incluido el maestro o maestra. La praxis crítica en el aula agrega al diálogo reflexivo el desarrollo un plan de acción 
colectivo para abordar los problemas que se tratan. El proceso de implementar el plan y evaluar la acción es también parte del proceso de aprendizaje (Duncan-Andrade y Morrell, 2008).

\section{Cultura escolar democrática}

La cultura escolar es mencionada en última instancia no porque su importancia sea menor que la del resto de las dimensiones, sino porque quizá sea el concepto más esquivo (DíezGutiérrez, 1999; Harris, 2018; Hinde, 2005; Schein, 2010). Quizá por esas ironías humanas de que lo más importante resulta, al fin y al cabo, lo menos aprehensible, la democracia en la cultura escolar da vida y significado a las otras dimensiones. Es lo que infunde sentido democrático y evita que su desarrollo sea una réplica mecánica, una aplicación pasiva de instructivos sin compromiso sincero.

El concepto de cultura surge de la antropología y hace referencia a patrones de sentido compartidos por una comunidad que se transmiten a través del tiempo y que se encarnan en símbolos e historias. Las normas, los valores, las creencias, las tradiciones, rituales, ceremonias y mitos que comparten las comunidades son algunos de sus elementos significativos (Geertz, 1973). La teoría de las organizaciones se hizo del concepto para comprender el funcionamiento de entidades como empresas, organismos públicos o incluso escuelas y llegó a demostrar que la cultura es el factor clave para entender su originalidad y las razones profundas de su funcionamiento, y para actuar sobre ellas cuando es necesario (Goldring, 2002; Schein, 2010).

La cultura de una organización está definida por elementos como los patrones de interacción, las normas, los valores comunes, la filosofía de grupo, los hábitos lingüísticos y de pensamiento, los símbolos y rituales, etc., pero es más que ello. Estos elementos unidos cumplen cuatro características básicas que nos acercan a una comprensión más profunda de la cultura: integración, profundidad, amplitud y estabilidad (Schein, 2010). Los elementos se integran en un paradigma más amplio con una coherencia no mentada y cuanto más profundamente estén incorporados en el grupo, cuanto menos tangible y visible sean, más nos acercaremos a la "esencia" de la cultura. Este patrón de integración cubre el funcionamiento del grupo, influye en todos los aspectos de la organización: las interacciones, los procesos, los objetivos, los entornos, etc. Ordena y aporta un sentido de identidad que da estabilidad y, por ello, no se abandona fácilmente. Al contrario, sobrevive a muchos miembros de la organización. Pero esta definición resalta el aspecto "estático". La cultura, además, puede ser entendida desde una definición "dinámica". Considerada desde esta perspectiva, es un proceso de creación permanente, un producto constante de las acciones e interacciones de la comunidad (Schein, 2010).

Las definiciones de cultura organizacional nos ayudan a entender mejor a las escuelas. Existen dos elementos que constituyen el "alma" del centro: su misión y sus creencias, valores y normas (Deal y Peterson, 2016). La misión, incluso cuando no sea consciente o no esté explicitada, funciona como fundamento de la organización, pues desencadena "fuerzas intangibles que inspiran a los maestros a enseñar, a los líderes escolares a liderar, a los niños a aprender y a los padres y la comunidad a tener confianza y fe en su escuela" (Deal y Peterson, 2016, p. 70).

Incluso cuando no sean puestas en común, todos tenemos una razón de actuar, sea esta autónoma o impuesta, sea esta común o fragmentada. Cuando es compartido, el propósito 
da sentido a la actividad escolar, otorga dirección y criterio para la toma de decisiones, motiva y genera compromiso entre los participantes. El segundo punto fundamental para considerar la cultura en las escuelas son los valores, creencias, normas y asunciones básicas arraigadas en los participantes. Se trata de los criterios éticos que ordenan la convivencia en la escuela y orientan la acción. A diferencia del propósito o misión, no son un objetivo por conseguir, sino un criterio para el accionar cotidiano.

En su aspecto estático, la cultura escolar también funciona como un "esquema de comportamiento" arraigado que da estabilidad y previsibilidad a las tareas cotidianas del centro (Deal y Peterson, 2016). Mas en su aspecto dinámico, es construida y amoldada permanentemente a través de las interacciones con otros y de las reflexiones de las propias personas y grupos sobre sus circunstancias. También en esta definición comparte rasgos con la cultura organizacional, pues se crea en las interacciones entre la comunidad educativa, pero estas no surgen de la nada, sino que son guiadas por la cultura escolar. Introducir un cambio en este ciclo es siempre difícil, pero es posible y permite entonces romper la inercia de reproducción (Hinde, 2005).

Comprender la cultura escolar nos ayuda a entender por qué las medidas tomadas a favor de la justicia social o de la democracia en las escuelas no siempre funcionan como esperamos. Una buena cantidad de investigaciones (p. ej., Mabovula, 2009; Mncube y Mafora, 2013; Olivo Pérez, Alaníz Hernández y Reyes García, 2011; Sapon-Shevin, 2013) analizan iniciativas adoptadas a favor de la democracia o de la justicia social en las escuelas y comprueban que en su puesta en práctica la participación no es genuina o que la justicia social se interpreta de manera restrictiva. Es que la democracia no es un cúmulo de procedimientos, ni un mero mecanismo para escoger la forma de organizarse o los contenidos del currículo. Y la justicia social no es una distribución mecánica de beneficios educativos ni un respeto impuesto a la diversidad cultural. La democracia y la justicia social son más que ello. Ambas se alimentan, en primer lugar, de los significados asumidos por la comunidad escolar y construidos en sus interacciones cotidianas y solo después de las iniciativas que se adoptan a su favor.

Por ello, las escuelas que trabajan por la democracia y la justicia social, antes que nada, hacen realidad los valores de la democracia y de la justicia social en sus interacciones cotidianas de una manera propia, coherente con su identidad de grupo. Crean una cultura escolar que encuentra y construye sentidos en torno a la democracia y a la justicia social. Para ello atienden tanto al aspecto dinámico como al aspecto estático de la cultura escolar.

Desde el aspecto dinámico, estas escuelas dan importancia a la construcción de grupo, a la creación de un sentido de identidad y pertenencia, de manera que la tarea educativa no es lo único que une a la comunidad educativa, sino que existe un vínculo emocional. Para ello, organizan encuentros que no son requeridos estrictamente por la labor educativa, pero que permiten reunirse, conocerse y disfrutar de la compañía mutua: fiestas escolares, reuniones docentes, actividades entre distintos grados y niveles educativos, etc.

Esto es coherente con los resultados de diversas investigaciones, pues está comprobado que los estudiantes asocian su participación en la escuela antes con las relaciones interpersonales, la confianza y las interacciones que con la participación en los procesos de decisión en sí mismos. En efecto, es este sentimiento el que conduce a una mayor implicación en los procesos de participación (John-Akinola et al., 2014). Por ello, Simó, Parareda y Domingo (2016) sostienen que la democracia se vincula sobre todo con el "sentirse partícipe de" y con las relaciones interpersonales. 
Estas escuelas entablan las interrelaciones de tal manera que todos pueden sentir que su participación es importante y que el grupo es distinto porque él o ella está allí también (Arendt, 2014). Estas escuelas construyen las interrelaciones cotidianas desde la escucha mutua, hay espacio para dar las propias opiniones y se reconocen las aportaciones de niños y niñas, de familias, del profesorado y de no docentes. De esta manera, la motivación para actuar, para adoptar una actitud proactiva ante los asuntos escolares, surge de manera "espontánea”.

En el aspecto estático de la cultura, estas escuelas prestan atención a la misión del centro y a sus valores más básicos. Reflexionan acerca de la educación que construyen y la que quieren. Definen de manera participativa la misión y el propósito de la escuela, de manera que existe debate acerca del sentido último del trabajo en el centro y de la relación entre las actividades que se realizan y esta misión compartida. Estas comunidades educativas reflexionan acerca de sus acciones cotidianas y se cuestionan a sí mismas. Pero también se preguntan en qué momento logramos aquello que queremos ser: juntan historias cotidianas en las que se sienten reflejadas y las narran a familias y amigos, incluso se las repiten a sí mismas, docentes y estudiantes, pues estas historias de "cómo somos y cómo queremos ser" proveen guía y motivación. Cuestionan de manera explícita sus valores y normas, los escogen y ordenan reflexionando sobre sus acciones y explicitando los criterios que guían la acción. Dentro de estos valores, asumen la redistribución y el reconocimiento como asuntos de justicia, no como obligaciones impuestas, y escogen hacer realidad la libertad, la igualdad y la solidaridad, más allá de las formas concretas en las que conciben. Organizan actividades para promover la familiaridad y resonancia de estos valores entre todos los miembros de la comunidad escolar. Aquí también la propia experiencia del centro y las narraciones sobre su historia, las narraciones que reflejan lo que para el centro es importante, constituye un aspecto esencial (Deal y Peterson, 2016).

Por último, estas escuelas aprenden de otras experiencias, de investigaciones, de recomendaciones, pero "dan vida" a la democracia y a la justicia social desde sus propias vivencias, desde su propia originalidad. El impulso de hacerlas realidad es interno al grupo, tal vez inspirado, pero nunca impuesto, de manera que el resultado es siempre una forma original, arraigada en lo más profundo y único de la comunidad escolar. De manera que estas escuelas construyen cotidianamente la democracia y la justicia social desde su personalidad particular.

\section{Conclusiones}

La relación entre democracia y justicia social suele darse más por supuesta que por explicada. Es habitual pensar que la democracia no puede ser genuina en una sociedad que no cumpla con condiciones mínimas de justicia, pero el modo concreto en que una y otra se necesitan y se complementan es menos claro. A partir de la teoría de Fraser (2000, 2008, 2012), este artículo explicitó una forma de entender esta relación y, a partir de ella, planteó dimensiones que ayudan a darle vida en las escuelas. Según esta perspectiva, sólo en condiciones de justicia es posible la participación en condiciones de igualdad, pues si existen condiciones materiales desiguales (redistribución injusta), estatus desigual (falta de reconocimiento) o si hay voces ausentes en los espacios de tomas de decisiones (problemas de representación), la participación no podrá darse de manera igualitaria. La participación democrática, a su vez, es necesaria para mejorar las condiciones de 
redistribución, de reconocimiento y de representación. En su relación mutua, justicia social y democracia van de la mano también en nuestras escuelas.

Las injusticias no se dan de manera "pura", sino que son siempre una interrelación. Diferenciarlas analíticamente ayuda a identificarlas y trabajar para revertirlas. En educación, los problemas de redistribución (arraigados en el dominio socioeconómico) nos remiten a dificultades en la asignación de recursos que impiden a las personas gozar por igual de las oportunidades educativas y beneficiarse luego por haber recibido una educación de calidad (Keddie, 2012; Lim y Tan, 2018; Lingard y Keddie, 2013; Lynch y Lodge, 2002; Power, 2012; Wang, 2016). Atender a esta dimensión para mejorar la práctica educativa significa partir de las condiciones socioeconómicas de los estudiantes y asignar los recursos de manera que todos y todas puedan aprovechar la educación que brinda la escuela. Significa también que los centros escolares se esfuerzan para que sus estudiantes alcancen una educación de calidad que les permita en el futuro insertarse socialmente en condiciones de igualdad con sus pares, independientemente de su situación socioeconómica inicial.

La falta de reconocimiento refiere a las injusticias arraigadas en el dominio sociocultural. Estudiantes que provienen de culturas no hegemónicas o cuyas identidades no se ven reflejadas en los cánones dominantes sufren una falta de reconocimiento y, como tal, una desigualdad en el estatus que les impide participar en igualdad con sus pares (Keddie, 2012; Lynch y Lodge, 2002; Power y Frandji, 2010; Sapon-Shevin, 2013). Al trabajar la dimensión del reconocimiento se admite que no existen valores o parámetros "normales", sino hegemónicos y que es preciso cuestionar estos supuestos para dejar al descubierto la invisibilización, el reconocimiento inadecuado o la subordinación. Esto permite, por un lado, cuestionar la educación hegemónica a partir de dar lugar a las experiencias y los paradigmas de conocimiento no dominantes, y por otro, entablar las relaciones cotidianas desde la inclusión y el respeto mutuo, viviendo la diversidad como experiencia cotidiana.

En términos de organización, el gobierno escolar es bisagra entre la justicia social y democracia, pues es un asunto de justicia si lo entendemos como representación: si la comunidad educativa no está representada en los órganos de decisión escolar en toda su diversidad, el resultado de las decisiones excluirá a algunas personas y colectivos. Pero, a la vez, a través de la dimensión de gobernanza se promueven formas más participativas y coordinadas de gobierno y gestión escolar. En este sentido, más relacionado con la democracia, se abre a la participación de toda la comunidad educativa, de estudiantes, docentes, familias, no docentes, incluso vecinos o miembros de la comunidad en general. Esta apertura crea los medios para que se puedan llevar adelante reclamos de justicia en la escuela. Esta dimensión fomenta nuevas formas de participación, de coordinación y colaboración entre los miembros de la comunidad educativa para una gestión más dinámica y horizontal (Santizo Rodall, 2011; Subirats, 2009). Prácticas como el liderazgo distribuido y la gestión en red son de ayuda para fomentar las iniciativas de los miembros, abrir la participación y trascender los límites del centro cooperando con entidades cercanas a la escuela.

Las dimensiones de redistribución, reconocimiento y gobernanza, aunque profundamente relacionadas, pueden entenderse como analíticamente diferenciadas, pero con las dimensiones de currículum escolar y de cultura democrática no sucede lo mismo. Aunque tienen una entidad propia que amerita tratarlas de manera independiente, están profundamente atravesadas por el resto. El currículum, por ejemplo, requiere cumplir con 
las dimensiones de la justicia y también ser definido (en términos generales) por la comunidad. En su puesta en práctica (en la escuela y en el aula) se desarrolla como una construcción participativa con el estudiantado que fomenta la reflexión crítica y la acción comprometida. A la vez, tanto democracia como justicia social son contenidos curriculares en sí mismos, pues es preciso enseñarlos como la conquista histórica que son y que necesitan seguir siendo si queremos que mantengan su fuerza.

La cultura, por último, nos permite poner sobre la mesa lo más importante: la democracia y la justicia social no son conceptos abstractos para implantar en las escuelas. Son construcciones concretas en su día a día y tanto mejores cuanto más incorporadas estén en su cultura escolar, según los rasgos propios de la comunidad educativa o, en otras palabras, la "personalidad" de cada escuela. Pues los sentidos y significados, las normas compartidas y los patrones de interrelación que se correspondan con formas justas y democráticas darán fuerza, espontaneidad y genuinidad a las iniciativas.

Esperamos que estas dimensiones ayuden a hacer frente al enorme desafío que significa construir escuelas más justas y democráticas. Las escuelas tienen mucho por hacer en este camino, pero para finalizar, es importante resaltar que no es total su responsabilidad. Por ello la organización en red, la apertura a la participación, son necesarias para reclamar una redistribución más justa y un reconocimiento de distintos saberes y formas de conocimiento por parte del sistema educativo. Comunidades cuyo compromiso con la justicia social y la participación democrática trasciendan el centro escolar, se construyan con entidades del entorno y busquen cambiar las condiciones del sistema educativo que resultan injustas.

\section{Agradecimientos}

Este artículo ha sido desarrollado en el marco del proyecto financiado por el Plan Estatal $\mathrm{I}+\mathrm{D}+\mathrm{i}$ del Gobierno de España "La democracia en las escuelas como fundamento de una educación para la Justicia Social” (Ref. EDU2017-82688-P).

\section{Referencias}

Abowitz, K. K. (2011). Qualifying my faith in the common school ideal: A normative framework for democratic justice. Educational Theory, 60(6), 683-702. https://doi.org/10.1111/j.1741-5446.2010.00384.x

Aguilar Villanueva, L. F. (2006). Gobernanza y gestión pública. Fondo de Cultura Económica.

Apple, M. W. (2014). Official knowledge: Democratic education in a conservative age. Routledge.

Apple, M. W. (2018). Ideology and curriculum. Routledge.

Apple, M. W. (2019). The struggle for democracy in education: Lessons from social realities. Routledge.

Apple, M. W. y Beane, J. A. (1999). Escuelas democráticas. Morata.

Arendt, H. (2014). La condición humana. Paidós.

Ayers, W., Kumashiro, K., Meiners, E., Quinn, T. y Stovall, D. (2016). Teaching toward democracy 2e: Educators as agents of change. Routledge.

Beane, J. A. (1997). Curriculum integration: Designing the core of democratic education. Teachers College, Columbia University. 
Beane, J. A. (2013). A common core of a different sort: Putting democracy at the center of the curriculum. Middle School Journal, 44(3), 4-14. https://doi.org/10.1080/00940771.2013.11461850

Bergmark, U. y Westman, S. (2015). Co-creating curriculum in higher education: Promoting democratic values and a multidimensional view on learning. International Journal for Academic Development, 21(1), 28-40. https://doi.org/10.1080/1360144X.2015.1120734

Blackmore, J. (2016). Educational leadership and Nancy Fraser. Routledge.

Brough, C. J. (2012). Implementing the democratic principles and practices of student-centred curriculum integration in primary schools. The Curriculum Journal, 23(3), 345-369. https://doi.org/10.1080/09585176.2012.703498

Carr, W. (1998). The curriculum in and for a democratic society. Pedagogy, Culture \& Society, 6(3), 323-340. https://doi.org/10.1080/14681369800200044

Deal, T. E. y Peterson, K. D. (2016). Shaping school culture. Jossey-Bass.

Dewey, J. (1916/2004). Democracia y educación: Una introducción a la filosofía de la educación. Morata.

Díez-Gutiérrez, E. J. (1999). La estrategia del caracol. Un cambio cultural en una organización. OikosTau.

Duncan-Andrade, J. M. y Morrell, E. (2008). The art of critical pedagogy: Possibilities for moving from theory to practice in urban schools. Peter Lang.

Dworkin, R. M. (2006). Is democracy possible here? Principles for a new political debate. Princeton University Press.

Englund, T. (2015). Toward a deliberative curriculum? Nordic Journal of Studies in Educational Policy, 1, art. 26558. https://doi.org/10.3402/nstep.v1.26558

Feito, R. (2009). Escuelas democráticas. Revista de Sociología de la Educación, 2(1), 17-33.

Feu, J., Prieto, O. y Simó, N. (2016). ¿Qué es una escuela verdaderamente democrática? Cuadernos de Pedagogía, 465, 90-97.

Feu, J., Simó, N., Serra, C. y Canimas, J. (2016). Dimensiones, características e indicadores para una escuela democrática. Estudios Pedagógicos (Valdivia), 42(3), 449-465.

Feu, J., Serra, C., Canimas, J., Làzaro, L. y Simó, N. (2017). Democracy and education: A theoretical proposal for the analysis of democratic practices in schools. Studies in Philosophy and Education, 36, 647-661. https://doi.org/10.1007/s11217-017-9570-7

Feu, J., Simó, N., Serra, C., Canimas, J. y Lázaro, L. (2016). Elementos claves para una gobernanza democrática de la escuela: Dimensiones e indicadores. En J. Collet y A. Tort (Coord.), La gobernanza escolar democrática (pp. 101-126). Morata.

Fielding, M. (2012). Beyond student voice: Patterns of partnership and the demands of deep democracy. Revista de Educación, 359, 45-65.

Fraser, N. (2000). Rethinking recognition. New Left Review, 2(3), 107-120.

Fraser, N. (2008). La justicia social en la era de la política de identidad: Redistribución, reconocimiento y participación. Revista de Trabajo, 4(6), 83-99.

Fraser, N. (2012). Escalas de justicia. Herder Editorial.

Freire, P. (1970). Pedagogía del oprimido. Siglo XXI Editores.

Freire, P. (1993). Pedagogía de la esperanza: Un reencuentro con la pedagogía del oprimido. Siglo XXI Editores. 
Gay, G. (2013). Teaching to and through cultural diversity. Curriculum Inquiry, 43(1), 48-70. https://doi.org/10.1111/curi.12002

Geertz, C. (1973). The interpretation of cultures. Basic books.

Gimeno Sacristán, J. (1991). El currículo: Una reflexión sobre la práctica. Morata.

Gimeno Sacristán, J. (2010). ¿Qué significa el curriculum?. En J. Gimeno Sacristán (Comp.), Saberes e incertidumbres sobre el currículum (pp. 21-44). Morata.

Giroux, H. A. (2001). Theory and resistance in education: Towards a pedagogy for the opposition. Greenwood Publishing Group.

Goldring, L. (2002). The power of school culture. Leadership, 32(2), 32-35.

Guarro, A. (2002). Currículo y democracia. Octaedro.

Guarro, A. (2008). Competencias básicas: Currículum integrado y aprendizaje cooperativo. Investigación en la Escuela, 66, 29-42.

Gutmann, A. (1987). Democratic education. Princeton University Press

Harris, J. (2018). Speaking the culture: Understanding the micro-level production of school culture through leaders' talk. Discourse, 39(3), 323-334.

https://doi.org/10.1080/01596306.2016.1256271

Hinde, E. R. (2005). School culture and change: An examination of the effects of school culture on the process of change. Essays in Education, 12, 61-72.

Honneth, A. (1996). The struggle for recognition: The moral grammar of social conflicts. Mit Press.

Hopkins, N. (2014). The democratic curriculum: Concept and practice. Journal of Philosophy of Education, 48(3), 416-427. https://doi.org/10.1111/1467-9752.12088

Hytten, K. (2015). Ethics in teaching for democracy and social justice. Democracy and Education, $23(2)$, art. 1.

Jones, S., Harvey, M., Lefoe, G. y Ryland, K. (2014). Synthesising theory and practice: Distributed leadership in higher education. Educational Management Administration \& Leadership 42(5), 603-619. https://doi.org/10.1177/1741143213510506

Keddie, A. (2012). Schooling and social justice through the lenses of Nancy Fraser. Critical Studies in Education, 53(3), 263-279. https://doi.org/10.1080/17508487.2012.709185

Kumashiro, K. K. (2015). Against common sense: Teaching and learning toward social justice. Routledge.

Lim, L. (2011). Beyond logic and argument analysis: Critical thinking, everyday problems and democratic deliberation in Cambridge international examinations' thinking skills curriculum. Journal of Curriculum Studies, 43(6), 783-807.

https://doi.org/10.1080/00220272.2011.590231

Lim, L. y Tan, M. (2018). Meritocracy, policy and pedagogy: Culture and the politics of recognition and redistribution in Singapore. Critical Studies in Education, 1-17.

https://doi.org/10.1080/17508487.2018.1450769

Lingard, B. y Keddie, A. (2013). Redistribution, recognition and representation: Working against pedagogies of indifference. Pedagogy, Culture and Society, 21(3), 427-447. https://doi.org/10.1080/14681366.2013.809373

Lynch, K. y Baker, J. (2005). Equality in education: An equality of condition perspective. Theory and Research in Education, 3(2), 131-164. https://doi.org/10.1177/1477878505053298

Lynch, K. y Lodge, A. (2002). Equality and power in schools. Routledge. 
Mabovula, N. (2009). Giving voice to the voiceless through deliberative democratic school governance. South African Journal of Education, 29(2), 219-233.

McLaren, P., Ryoo, J. J., Crawford, J. y Moreno, D. (2010). Critical praxis. En C. A. Kridel (Coord.), Encyclopedia of curriculum studies (pp. 150-151). Sage. http://doi.org/10.4135/9781412958806.n90

Mills, C. y Gale, T. (2010). Schooling in disadvantaged communities. Springer.

Mills, M., McGregor, G., Baroutsis, A., Te Riele, K. y Hayes, D. (2016). Alternative education and social justice: Considering issues of affective and contributive justice. Critical Studies in Education, 57(1), 100-115. https://doi.org/10.1080/17508487.2016.1087413

Mncube, V. y Mafora, P. (2013). School governing bodies in strengthening democracy and social justice: parents as partners? Anthropologist, 15(1), 13-23. https://doi.org/10.1080/09720073.2013.11891288

Moliner, O., Traver, J. A., Ruiz, M. P. y Segarra, T. (2016). Estrategias que inciden en los procesos de democratización de la escuela. Una aproximación teórica. Revista Electrónica de Investigación Educativa, 18(2), 116-129.

Murillo, F. J. (2006). Una dirección escolar para el cambio: Del liderazgo transformacional al liderazgo distribuido. REICE. Revista Iberoamericana sobre Calidad, Eficacia y Cambio en Educación, 4(4), 11-24.

Murillo, F. J. y Hernández, R. (2011). Hacia un concepto de justicia social. REICE. Revista Iberoamericana sobre Calidad, Eficacia y Cambio en Educación, 9(4), 7-23.

Murillo, F. J. y Hernández, R. (2014). Liderando escuelas justas para la justicia social. Revista Internacional de Educación para la Justicia Social, 3(2), 13-32

Olivo Pérez, M. A., Alaníz Hernández, C. y Reyes García, L. (2011). Crítica a los conceptos de gobernabilidad y gobernanza: Una discusión con referencia a los consejos escolares de participación social en México. Revista Mexicana de Investigación Educativa, 16(50), 775-799.

Power, S. (2012). From redistribution to recognition to representation: Social injustice and the changing politics of education. Globalisation, Societies and Education, 10(4), 473-492. https://doi.org/10.1080/14767724.2012.735154

Power, S. y Frandji, D. (2010). Education markets, the new politics of recognition and the increasing fatalism towards inequality. Journal of Education Policy, 25(3), 385-396. https://doi.org/10.1080/02680930903576404

Rawls, J. (1971). A theory of justice. Harvard University Press.

Riddle, S. y Apple, M. W. (Eds.). (2019). Re-imagining education for democracy. Routledge.

Ruitenberg, C. (2009). Educating political adversaries: Chantal Mouffe and radical democratic citizenship education. Studies in Philosophy and Education, 28(3), 269-281. https://doi.org/10.1007/s1 1217-008-9122-2

Santizo Rodall, C. (2011). Gobernanza y participación social en la escuela pública. Revista Mexicana de Investigación Educativa, 16(50), 751-773.

Sapon-Shevin, M. (2013). La inclusión real: Una perspectiva de justicia social. Revista de Investigación en Educación, 11(3), 71-85.

Schein, E. H. (2010). Organizational culture and leadership. Jossey-Bass.

Simó, N., Parareda, A. y Domingo, L. (2016). Towards a democratic school. The experience of secondary school pupils. Improving Schools, 19(3), 181-196. https://doi.org/10.1177/1365480216631080 
Smit, H. H. y Oosthuizen, I. J. (2011). Improving school governance through participative democracy and the law. South African Journal of Education, 31, 55-73.

Straume, I. (2016). Democracy, education and the need for politics. Studies in Philosophy and Education, 35(1), 29-45. https://doi.org/10.1007/s11217-015-9465-4

Subirats, J. (2009). Gobernanza y educación. En VVAA, Educación y vida urbana, 20 años de ciudades educadoras (pp. 227-240). Asociación Internacional de Ciudades Educadoras.

Subirats, J. (2016). Notas sobre principios y estrategias de una gobernanza educativa y democrática de lo común en el cambio de época. En J. Collet y A. Tort (Coords.), La gobernanza escolar democrática. Más allá de los modelos neoliberal y neoconservador (pp. 65-83). Morata.

Taylor, C. (1994). Multiculturalism: Examining the politics of recognition. Princeton University Press.

Torres, J. (2006). Globalización e interdisciplinariedad: El currículum integrado. Ediciones Morata.

Vincent, C. (Ed.). (2020). Nancy Fraser, social justice and education. Routledge.

Wang, F. (2016). From redistribution to recognition: How school principals perceive social justice. Leadership and Policy in Schools, 15(3), 323-342. https://doi.org/10.1080/15700763.2015.1044539

Woods, P. A. y Woods, G. J. (2012). Degrees of school democracy: A holistic framework. Journal of School Leadership, 22(4), 707-732. https://doi.org/10.1177/105268461202200402

Young, I. M. (2011). Justice and the politics of difference. Princeton University Press.

\section{Breve CV de los autores}

\section{Guillermina Belavi}

Investigadora posdoctoral con un contrato de Formación del Personal Investigador (FPIUAM) obtenido por concurrencia competitiva. Miembro del grupo de investigación Cambio Educativo para la Justicia Social (GICE-UAM), de la Cátedra UNESCO en Educación para la Justicia Social, del Instituto de Derechos Humanos, Democracia, Cultura de Paz y No Violencia (DEMOSPAZ) y de la Red de Investigación sobre Liderazgo y Mejora de la Educación (RILME). Es Doctora en Educación por la Universidad Autónoma de Madrid y Licenciada en Ciencia Política por la Universidad Nacional de Rosario. Su área de interés es la filosofía política y educación, actualmente desarrolla su investigación en el marco del Proyecto I+D+I "La democracia en las escuelas como fundamento de una educación para la Justicia Social” (Ref: EDU2017-82688-P). ORCID ID: http://orcid.org/0000-0002-6471-7583. Email: guillermina.belavi@uam.es

\section{F. Javier Murillo}

Director de la Cátedra UNESCO en Educación para la Justicia Social de la Universidad Autónoma de Madrid, profesor Titular en Métodos de Investigación y Evaluación en Educación en la Universidad Autónoma de Madrid, coordinador del Grupo de Investigación Cambio Educativo para la Justicia Social (GICE), coordinador de la Red de Investigación sobre Liderazgo y Mejora de la Educación (RILME), coordinador general de la Red Iberoamericana de Investigación sobre Cambio y Eficacia Escolar (RINACE). Es director de REICE. Revista Iberoamericana sobre Calidad, Eficacia y Cambio en Educación, director de la Revista Iberoamericana de Evaluación Educativa, director de la Revista Internacional de Educación para la Justicia Social y secretario académico del 
Instituto de Derechos Humanos, Democracia y Cultura de Paz y No Violencia (DEMOSPAZ) de la UAM. Página web: https://fjaviermurillo.es. ORCID ID: https://orcid.org/0000-0002-8003-4133. Email: javier.murillo@uam.es 